\title{
Using Sports Wagering Markets to Evaluate and Compare Team Winning Streaks in Sports
}

\author{
R. Alan Bowman', Thomas Ashman², James Lambrinos ${ }^{1}$ \\ ${ }^{1}$ School of Management, Union Graduate College, Schenectady, NY, USA \\ ${ }^{2}$ Behavioral Sciences Collegium, Eckerd College, St. Petersburg, FL, USA \\ Email: lambrinj@uniongraduatecollege.edu
}

Received 15 July 2015; accepted 22 August 2015; published 25 August 2015

Copyright (C) 2015 by authors and Scientific Research Publishing Inc.

This work is licensed under the Creative Commons Attribution International License (CC BY). http://creativecommons.org/licenses/by/4.0/

(c) (i) Open Access

\begin{abstract}
Point spreads and money lines from sports wagering markets are used to evaluate the impressiveness of team streaks. Sports wagering data have previously been useful in assessing competitive balance in sports. Our approach was motivated by the amount of media scrutiny that accompanied the winning streak of the NBA's Miami Heat and the point streak of the NHL's Chicago Blackhawks which occurred simultaneously for the most part in 2013. The topic came to the forefront again with the 2014-2015 winning streak of the Atlanta Hawks. Three streaks are highlighted in our paper. The length of the streaks, the quality of the competition, injuries, and a variety of other factors were mentioned in the media but the discussion was limited to subjective opinions as no way of properly weighing relative influence of the factors was identified. Wagering markets provide an excellent source of information for making these judgments. Several complementary measures are described and the most impressive team streaks within and across professional baseball, basketball, football, and ice hockey are identified.
\end{abstract}

\section{Keywords}

Winning Streaks, Sports Wagering Markets, Baseball, Basketball, Football, Hockey

\section{Introduction}

In 2013, the Miami Heat in the NBA and Chicago Blackhawks in the NHL were simultaneously in the middle of long streaks (a 26-game winning streak for the Heat and a 24-game points streak for the Blackhawks). This fueled a very active (albeit brief) debate in the media about which streak was more impressive. Many factors were 
brought up in the discussions besides just the obvious length of the streak including the quality of competition, home vs. away games, travel, back-to-back games, injuries, and many others including the inherent difficulty of comparing streaks in one sport vs. another. In the 2014-15 NBA season, the Atlanta Hawks won 19 straight games. Unlike the Heat (with Lebron James, Dwyane Wade, and Chris Bosh), the Hawks were not perceived for the most part to be a dominating team-their streak was a bit more of a surprise. Should one consider this in judging the impressiveness of the Hawks' streak and possibly even conclude that the Hawks' streak was more impressive than the Heat's even though it was shorter? The NBA's Eastern Conference has recently been significantly weaker than the Western Conference. Did either the Heat or the Hawks simply beat up on Eastern patsies? Should this be taken into account and, if so, how? Is there any way to legitimately compare streaks in the same sport, much less across sports? Although this topic has generated considerable public interest and discussion, we are unaware of any serious treatment of it in the literature. We seek to provide an objective, unbiased, and rigorous quantitative approach for assessing the impressiveness of sports streaks that incorporates as many relevant factors as possible and to use our approach to evaluate and compare past winning streaks both within and across professional sports. We present our approach and results in this paper.

Since Mottley [1] suggested using OR techniques to analyze sports over 50 years ago, there has been a growing interest in doing so. Wright [2] provides an excellent summary, categorization, and analysis of articles that have addressed a wide variety of issues that fall under this broad umbrella in the last 50 years. Certainly one reason for the growing popularity of this field is that sports are entertaining. This has important consequences for the broader OR field. First, OR approaches can enhance the entertainment value of sports by providing analytical insight into issues that are otherwise not available. This in turn enhances the visibility of OR. Also, the inherent entertainment value of sports makes them ideal for introducing students to OR techniques and thought processes. The use of OR in the classroom is widespread and growing. Cochran [3] provides a good introduction to this topic, focusing on articles that were included in a special issue of INFORMS Transactions on Education devoted to the topic of Sports in the OR Classroom. We believe our paper addresses an entertaining topic in a rigorous analytical fashion that provides insights that will enhance the entertainment value. We also think this topic and our approach are very well suited for use as an example in the OR classroom.

Our approach utilizes gambling line information. We believe gambling line information is ideal because it needs to take into account as many factors as possible so that gamblers will not be able to identify and exploit factors that are not considered (known as inefficiencies) in order to systematically make profits. As we began exploring ways to use this information, we realized that there are qualitative, perhaps even philosophical, issues to consider as well as quantitative factors. It became clear that there could be no single measure on which everyone would agree. We therefore developed several measures that provide different and complementary insights. We will briefly discuss all the measures we developed but we will focus our attention on two measures that most directly address the issues brought up in the media discussion of the streaks of the Heat and the Blackhawks and in our own considerations of those and other streaks such as the Hawks' recent streak. We then present results from computing these two measures for all actual streaks in the following professional sports and seasons for which we have comprehensive gambling line data:

- Baseball (1999 through 2014)

- Basketball (1990-91 through 2014-15)

- Football (1985-86 through 2014-15)

- Hockey (2005-6 through 2014-15)

Note that hockey started using shootouts in 2005-6 so that each game has a winner from that season forward. Winning streaks and points streaks, as well as the nature of the gambling line information, are very different prior to that season. For ease of comparability with the other sports, we have limited our treatment of hockey to data from 2005 forward. Also, in computing our results, we restrict our attention to regular season streaks and do not consider streaks that cross seasons. The approach for computing the measures can, for the most part, be extended to consider other sports and other types of streaks as long as gambling line data are available.

We will present the most impressive streaks according to our measures during the time frames shown above for each sport. One of the nice features of our approach, however, is that it allows streaks to be compared across different sports. To that end, we will present the most impressive streaks across all four sports. Our measures also allow insights into why streaks occur more frequently in some sports than others so we will discuss those issues as well. Finally, we will compare the streaks of the Heat, the Blackhawks, and the Hawks and offer our take on which one was most impressive, all things considered. 


\section{Why Use Gambling Lines?}

Streaks have always fascinated sports fans and, not surprisingly, they have received considerable attention in the literature. We believe that previous approaches for evaluating streaks (team or individual), however, have never dealt specifically and effectively with the types of issues raised in the media debate comparing the Heat's and Blackhawks' streaks. To see why we think this, consider what is arguably the most famous streak in all of sports-Joe DiMaggio's 56 game hitting streak in baseball. This streak has been analyzed in many outlets using various approaches (see, for example, Arbesman and Strogatz [4] and Rockoff and Yates [5]). The primary goal has always been to estimate the probability that a streak of that length would occur.

No approach, however, considers the actual games that the Yankees played during the streak and all the factors in those games that might affect the impressiveness of that streak. For example, who were the opposing pitchers, were the games at home or away, were the stadiums ones in which DiMaggio had success in the past, which Yankee hitter batted in front of and behind DiMaggio in that particular game, etc.?

Similarly, approaches for evaluating team streaks have focused on the likelihood that streaks of certain lengths would occur. Note that, with this focus, all streaks of the same length in a particular sport would be considered equivalent, essentially ignoring all the other factors that might differentiate one streak from another. A key component of much of this type of research has been to try to assess whether there is "streakiness" at all or whether the streaks observed are consistent with random variation (associated with sometimes quite sophisticated models). We think this research has been very interesting and valuable but does not deal directly with the issues raised in the media debate about the streaks of the Heat and the Blackhawks. Therefore, we established two criteria for our analysis of team streaks. First, we wanted to use actual games played in all of our measures. Second, we wanted to take into account as many factors as possible that were present during those actual games that would affect the likelihood that each team would win. These factors would include all the factors that fans might bring up in discussing and comparing actual streaks (quality of competition, home vs. away games, travel, back-to-back games, injuries, illnesses, starting pitchers or goalies, weather, etc.). This led to our decision to use gambling line data to evaluate the impressiveness of win streaks. Gambling lines theoretically will comprehensively consider all game-specific factors (more on this later) without specifically quantifying the effect of each factor. Importantly, these factors can change greatly from game to game, and properly recognizing that fact is crucial for comparing streaks in terms of their impressiveness. For example, at the start of the 2013 season, a five game winless streak by the Green Bay Packers in weeks 9-13 would have been considered quite unlikely. They were considered at the start of the season to be one of the stronger teams in the NFL. Prior to the injury to Packers quarterback Aaron Rodgers, the Green Back Packers were favored in seven of eight games. They won five of the seven before the injury. After his injury, the Packers were favored in only two of five games against weak opponents and did not win a single game during this period. This winless streak of five games against weak opponents would have been rather surprising at the start of the season but was not that surprising given the injury situation for Aaron Rodgers. Furthermore, winning streaks by other teams that included beating the Packers with Aaron Rodgers would be far more impressive than those that included beating the Packers without Aaron Rodgers. The gambling lines for either set of games properly reflect this fact.

An alternative approach that would satisfy our two criteria would be to develop a statistical model that explicitly includes as many factors as possible and apply that model to the factors as they existed for each actual game that was part of the streak. Historically, this alternative has not been feasible but, with the proliferation of data that are being gathered and made accessible, this might very well be feasible in the future. If such attempts are successful, our paper should prove quite helpful in providing a systematic approach for incorporating the individual game probabilities from the statistical models (in place of the ones we get from the gambling lines) into several complementary measures that together yield a range of insights.

At the heart of our measures is the use of gambling lines to provide us with the probability (prior to each game) that each team will win the game. The use of gambling lines in this manner has a relatively long and extensive history (see, for examples Peel and Thomas [6] [7]). All studies (such as ours) that use gambling lines as predictors of game outcomes are essentially assuming that the markets are at least reasonably efficient. This is closely tied to our claim that the gambling lines theoretically will comprehensively consider the game-specific factors that we believe are crucial to consider in evaluating team winning streaks. The idea is that, if the gambling lines do not properly include one or more these factors, informed bettors could exploit their knowledge of these factors (the market inefficiency) to consistently make money. There is also a long stream of sports research 
that examines whether sports betting markets are efficient. Although the literature on this subject is nuanced and complex, the broad conclusion is that the gambling lines generally provide unbiased estimators of game outcomes and market inefficiencies are corrected over time (see, for example, Sauer [8], and Gandar, Zuber, and Dare [9]).

\section{Measures for Assessing Winning Streaks}

The first measure we will describe is the most straightforward. We will refer to this simply as the streak probability (SP). The first step to compute SP is to convert the gambling line for each actual game in the streak to the probability of each team winning the game. The favorite win probability is $\frac{\frac{f}{100+f}}{\frac{f}{100+f}+\frac{100}{100+u}}$, where $f$ is the absolute value of the favorite money line and $u$ is the underdog money line. Of course, the underdog win probability is 1-the favorite win probability. In the cases of the NFL and NBA, the gambling line historical information that was most available came in the form of spreads. This required a preliminary step to convert the spread into the probability that each team would win. For evaluating hockey point streaks, it was necessary to first convert the probability each team would win to the probability that each team would win in regulation (interestingly, favored teams get a larger percentage of their wins in regulation than do underdogs), and the probability each team gets a point is one minus the probability the other wins in regulation. Details on how we accomplished these preliminary steps (and the actual numeric models) are available on request.

$\mathrm{SP}$, the probability that the team would win all the games in the streak, is simply the product of the individual game win probabilities:

$\mathrm{SP}=\prod_{i=1}^{n} p_{i}$, where $p_{i}=$ probability that the team with the winning streak would win game $i$ of the actual streak (computed from the money line for that game as shown above).

Note that, since we use the gambling line for each game at the time of the game, we are not computing the probability that the team will win all the games in the streak prior to the streak (or prior to the season) nor are we assuming independence. Instead, we are multiplying the conditional probabilities that they win each game (conditioned on all the factors known and taken into consideration in the gambling line prior to each game).

SP considers the team that actually had the winning streak in the computations. As we will discuss shortly, it might be desirable to have a measure that is independent of the team involved but includes all the other factors in the games. Therefore, we developed a second measure, which we will refer to as the average team streak probability (ATSP). ATSP requires the win probability for each game in the streak to be adjusted to what it would be if the average team in the league were playing each game rather than the team that actually had the streak. How this is done is shown in the Appendix. ATSP is then the product of the probabilities that the average team would win each of the games in the streak:

ATSP $=\prod_{i=1}^{n} a t p_{i}$, where $a t p_{i}=$ probability that the average team in the league would win game $i$ of the actual streak (computed using the money line for that game as shown above and the adjustment procedure described in the appendix).

In terms of evaluating streaks, both SP and ATSP take into consideration the quality of the opponents played during the streak, whether the games were played home or away, and any other game-specific factors that gamblers would consider such as the ones we identified in our earlier discussion. SP considers the quality of the team that actually had the streak whereas ATSP does not. In that sense, a good team that is highly regarded in the gambling lines would end up with a higher probability of the streak, hence the streak would be considered less impressive. One could argue that this should be true and that a long streak from a not-so-great team is more impressive but one could also argue that the measure of the impressiveness of the streak should be independent of the team that had the streak. For these reasons, we believe both measures have value and present results from both.

To illustrate these concepts and introduce an approach for decomposing SP and ATSP into components that we believe provide many useful insights, we present results for a couple of extreme winning streaks in the NFL. From 11/19/2006 through 12/24/2006, the Tennessee Titans won 6 games in a row. In 2007, the New England 
Patriots won all of their regular season games (16 games in a row). The SP for the Titans' streak is 0.0009579 compared to 0.04142827 for the Patriots' streak. This would indicate the Titans' streak is far more impressive. ATSP tells a different story. The ATSP is 0.00000285 for the Patriots' streak compared to 0.00944903 for the Titans'. So which streak is really more impressive? We can break it down further for more insights. Note that the geometric mean of the win probabilities for the Titans during their streak (easily computed for any streak as the streak probability raised to the power of the reciprocal of the streak length) is 0.3140 . In other words, if they had a 0.3140 chance of winning each of their 6 games, their streak would have a probability of 0.00095790 , the same as their SP. We will denote this geometric mean as GMSP. On the other hand, GMSP for the Patriots' 16 games was 0.8196 . This seems to indicate that the Patriots were simply a far more impressive team than the Titans. It could be, however, that the Patriots played a much easier schedule. We can break that component out as well. The geometric mean of the win probabilities for the average team in the league playing the Titans' 6 games was 0.4598 . We denote this geometric mean as GMATSP. For the Patriots' 16 games, the GMATSP was 0.4502. Both teams faced difficult schedules during their streaks and, in fact, the Patriots' schedule was slightly more difficult.

To enable further insights to be obtained, we now introduce two new concepts-the schedule factor and the dominance factor. If we define the schedule factor for any streak as SF = GMATSP/.5 and the dominance factor for any streak as DF $=$ GMSP/GMATSP, we can represent SP and ATSP as:

$$
\mathrm{SP}=(\mathrm{DF} \times \mathrm{SF} \times 0.5)^{L} \text { and } \mathrm{ATSP}=(\mathrm{SF} \times 0.5)^{L} \text {, where } L=\text { the streak length. }
$$

The larger the dominance factor the more impressive the team was deemed to be and the larger the schedule factor the easier the games in the streak were deemed to be (with 1.0 being average for both factors so that, if both factors were average, both probabilities would be the same as evaluating a series of coin flips). The Patriots had a dominance factor of 1.820 and a schedule factor of 0.900 during their streak. The Titans had a dominance factor of 0.683 and a schedule factor of 0.920 during their streak.

We think most people would say that the Patriots streak was more impressive as the reason their SP was not that impressive was because they were heavily favored in most of their games. In that sense, we think most people will find ATSP to be a better measure than SP. For the most part, we agree. On the other hand, the Titans were not deemed to be a very good team and they won 6 games in a row against a difficult schedule. They were underdogs in all 6 games and heavy underdogs in 2 of the 6 . This is impressive in its own right and SP reflects this. We believe SP and ATSP are both useful and provide complementary insights.

Note that both the SP and ATSP measures for a streak reflect only the probabilities for that specific streak and not a generic streak of that length. A consequence of this is that these two measures do not consider the frequency of opportunities for streaks (because each streak has only one opportunity). Sports with more games, however, clearly have more opportunities for streaks. As we mentioned earlier, much of the literature on streaks has been interested in the likelihood of a generic streak of a specific length and have properly considered the relative availability of opportunities, sometimes with quite sophisticated models. We have not seen gambling lines incorporated in such analyses so we note briefly here that it is possible to do so (and thereby consider actual games and actual factors involved in games) by combining the approach we have described here with computer simulations of actual seasons. We have done so and can provide details of both the approach and the results upon request. We note, however, that this approach considers actual games and factors across an entire season and how those affect generic streak probabilities for the season; it does not directly focus on the games and factors specifically present during the actual streak. We do not believe them to be as directly useful for our objectives here as the SP and ATSP measures.

\section{Empirical Results}

We first present results for SP and ATSP for the time frames analyzed that we previously identified. Table 1 and Table 2 show the five most impressive streaks for each sport as well as overall (obtained by computing SP and ATSP for every single win streak in each sport for their respective time frames and ranking them). Sports fans will, of course, enjoy looking over the specific streaks and thinking about the actual teams involved to see if the measures match up to their memories and opinions. We will focus here on some overall observations and we will close by comparing the streaks of the Heat, the Blackhawks, and the Hawks since that motivated the investigation in the first place. 
Table 1. Most impressive streaks: Winning streak probability (SP).

\begin{tabular}{|c|c|c|c|c|c|c|c|c|}
\hline & \multicolumn{3}{|c|}{ Streak Start } & \multicolumn{3}{|c|}{ Streak End } & Games & Probability (SP) \\
\hline Portland (NBA) & 12 & 3 & 2007 & 12 & 30 & 2007 & 13 & 0.00000574 \\
\hline San Diego (MLB) & 6 & 18 & 1999 & 7 & 2 & 1999 & 14 & 0.00003763 \\
\hline Houston (NBA) & 11 & 1 & 1996 & 12 & 15 & 1996 & 19 & 0.00006462 \\
\hline Tampa Bay (MLB) & 6 & 9 & 2004 & 6 & 22 & 2004 & 12 & 0.00006865 \\
\hline Chicago (NHL Points) & 1 & 19 & 2013 & 3 & 6 & 2013 & 24 & 0.00007414 \\
\hline \multicolumn{9}{|l|}{ MLB } \\
\hline San Diego & 6 & 18 & 1999 & 7 & 2 & 1999 & 14 & 0.00003763 \\
\hline Tampa Bay & 6 & 9 & 2004 & 6 & 22 & 2004 & 12 & 0.00006865 \\
\hline Atlanta & 7 & 26 & 2013 & 8 & 9 & 2013 & 14 & 0.00016572 \\
\hline Colorado & 6 & 4 & 2009 & 6 & 14 & 2009 & 11 & 0.00018205 \\
\hline Baltimore & 9 & 7 & 1999 & 9 & 22 & 1999 & 13 & 0.00020362 \\
\hline \multicolumn{9}{|l|}{$\underline{\text { NBA }}$} \\
\hline Portland & 12 & 3 & 2007 & 12 & 30 & 2007 & 13 & 0.00000574 \\
\hline Houston & 11 & 1 & 1996 & 12 & 15 & 1996 & 19 & 0.00006462 \\
\hline New York & 3 & 18 & 2013 & 4 & 9 & 2013 & 13 & 0.00020393 \\
\hline Atlanta & 12 & 27 & 2014 & 1 & 31 & 2015 & 19 & 0.00026859 \\
\hline Houston & 3 & 3 & 1991 & 4 & 6 & 1991 & 17 & 0.00028154 \\
\hline \multicolumn{9}{|l|}{$\underline{\text { NFL }}$} \\
\hline Pittsburgh & 9 & 26 & 2004 & 1 & 2 & 2005 & 14 & 0.00018249 \\
\hline New England Patriots & 10 & 5 & 2003 & 12 & 27 & 2003 & 12 & 0.00080506 \\
\hline Tennessee Titans & 11 & 19 & 2006 & 12 & 24 & 2006 & 6 & 0.00095790 \\
\hline San Diego & 9 & 20 & 1987 & 11 & 15 & 1987 & 8 & 0.00173198 \\
\hline Indianapolis Colts & 9 & 13 & 2009 & 12 & 17 & 2009 & 14 & 0.00218152 \\
\hline \multicolumn{9}{|l|}{$\underline{\text { NHL (Winning) }}$} \\
\hline Ottawa & 1 & 14 & 2010 & 2 & 4 & 2010 & 11 & 0.00015600 \\
\hline Columbus & 3 & 18 & 2015 & 4 & 4 & 2015 & 9 & 0.00034126 \\
\hline Pittsburgh & 3 & 2 & 2013 & 3 & 30 & 2013 & 15 & 0.00037185 \\
\hline New Jersey & 3 & 28 & 2006 & 4 & 18 & 2006 & 11 & 0.00058317 \\
\hline Phoenix & 12 & 28 & 2006 & 1 & 9 & 2007 & 7 & 0.00078838 \\
\hline \multicolumn{9}{|l|}{ NHL (Points) } \\
\hline Chicago & 1 & 19 & 2013 & 3 & 6 & 2013 & 24 & 0.00007414 \\
\hline San Jose & 2 & 21 & 2008 & 4 & 1 & 2008 & 20 & 0.00045401 \\
\hline Columbus & 3 & 18 & 2015 & 4 & 11 & 2015 & 13 & 0.00059309 \\
\hline Columbus & 2 & 26 & 2013 & 3 & 22 & 2013 & 12 & 0.00085031 \\
\hline Washington & 3 & 23 & 2006 & 4 & 5 & 2006 & 7 & 0.00093308 \\
\hline
\end{tabular}


Table 2. Most impressive streaks: Average team winning streak probability (ATSP).

\begin{tabular}{|c|c|c|c|c|c|c|c|c|}
\hline & \multicolumn{3}{|c|}{ Streak Start } & \multicolumn{3}{|c|}{ Streak End } & Games & Probability (ATSP) \\
\hline Miami (NBA) & 2 & 3 & 2013 & 3 & 25 & 2013 & 27 & 0.00000024 \\
\hline Houston (NBA) & 11 & 1 & 1996 & 12 & 15 & 1996 & 19 & 0.00000044 \\
\hline New England (NFL) & 9 & 9 & 2007 & 12 & 29 & 2007 & 16 & 0.00000285 \\
\hline Houston (NBA) & 1 & 29 & 2008 & 3 & 16 & 2008 & 22 & 0.00000433 \\
\hline Seattle (NBA) & 1 & 21 & 1996 & 3 & 18 & 1996 & 19 & 0.00000467 \\
\hline \multicolumn{9}{|l|}{ MLB } \\
\hline Oakland & 8 & 13 & 2002 & 9 & 4 & 2002 & 20 & 0.00000991 \\
\hline Atlanta & 4 & 16 & 2000 & 5 & 2 & 2000 & 15 & 0.00005043 \\
\hline Seattle & 5 & 23 & 2001 & 6 & 8 & 2001 & 15 & 0.00006312 \\
\hline Baltimore & 9 & 7 & 1999 & 9 & 22 & 1999 & 13 & 0.00009513 \\
\hline San Diego & 6 & 18 & 1999 & 7 & 2 & 1999 & 14 & 0.00011538 \\
\hline \multicolumn{9}{|l|}{$\underline{\text { NBA }}$} \\
\hline Miami & 2 & 3 & 2013 & 3 & 25 & 2013 & 27 & 0.00000024 \\
\hline Houston & 11 & 1 & 1996 & 12 & 15 & 1996 & 19 & 0.00000044 \\
\hline Houston & 1 & 29 & 2008 & 3 & 16 & 2008 & 22 & 0.00000433 \\
\hline Seattle & 1 & 21 & 1996 & 3 & 18 & 1996 & 19 & 0.00000467 \\
\hline L.A. Lakers & 2 & 4 & 2000 & 3 & 13 & 2000 & 19 & 0.00000570 \\
\hline \multicolumn{9}{|l|}{$\underline{\text { NFL }}$} \\
\hline New England & 9 & 9 & 2007 & 12 & 29 & 2007 & 16 & 0.00000285 \\
\hline Pittsburgh & 9 & 26 & 2004 & 1 & 2 & 2005 & 14 & 0.00005137 \\
\hline Indianapolis & 9 & 13 & 2009 & 12 & 17 & 2009 & 14 & 0.00007558 \\
\hline Green Bay & 9 & 8 & 2011 & 12 & 11 & 2011 & 13 & 0.00013417 \\
\hline Washington & 9 & 1 & 1991 & 11 & 17 & 1991 & 11 & 0.00017511 \\
\hline \multicolumn{9}{|l|}{ NHL (Winning) } \\
\hline Pittsburgh & 3 & 2 & 2013 & 3 & 30 & 2013 & 15 & 0.00002684 \\
\hline Washington & 1 & 13 & 2010 & 2 & 7 & 2010 & 14 & 0.00015849 \\
\hline Ottawa & 1 & 14 & 2010 & 2 & 4 & 2010 & 11 & 0.00022054 \\
\hline San Jose & 2 & 21 & 2008 & 3 & 14 & 2008 & 11 & 0.00032518 \\
\hline Buffalo & 10 & 4 & 2006 & 10 & 26 & 2006 & 10 & 0.00041916 \\
\hline \multicolumn{9}{|l|}{ NHL (Points) } \\
\hline Chicago & 1 & 19 & 2013 & 3 & 6 & 2013 & 24 & 0.00002269 \\
\hline San Jose & 2 & 21 & 2008 & 4 & 1 & 2008 & 20 & 0.00020611 \\
\hline Detroit & 3 & 9 & 2006 & 4 & 17 & 2006 & 20 & 0.00063915 \\
\hline Pittsburgh & 1 & 13 & 2007 & 2 & 18 & 2007 & 16 & 0.00065514 \\
\hline Vancouver & 12 & 8 & 2010 & 1 & 11 & 2011 & 17 & 0.00085407 \\
\hline
\end{tabular}


We first note that the NFL does not have any of the top five overall streaks as measured by SP but the Patriots' 2007 unbeaten season does make the top 5 ATSP results. Since we do not consider streaks across seasons, the NFL is at a disadvantage not only because the maximum possible streak is much shorter but also because a larger percentage of streaks will end (because the season ends) without a loss. Perhaps there is a reasonable adjustment for streaks that are active at season's end that could be made. There is another (and we think more interesting) issue involved. This issue can also be seen in the fact that the NBA has 4 of the top 5 ATSP results (the Patriots' being the $5^{\text {th }}$ ) whereas the SP streaks are more evenly spread across the NBA, NHL, and MLB. To understand why this occurred, we computed the dominance factors and the schedule factors described in the previous section for all streaks of at least 10 games in each sport. Summarized results are presented in Table 3 . We see that the average dominance factors are much higher in basketball and football than in hockey and baseball. The average schedule factors are higher than one in all sports, indicating that streaks are more likely during portions of the schedule that are easier, which makes sense. The average schedule factor is much higher for basketball than the other sports. We can see that basketball has more frequent long streaks than football primarily because there are more games per season and more than baseball and hockey primarily because there are more frequently teams that are more dominant and, to a lesser extent, portions of the schedule that are weaker. One note for sports fans is that the New England Patriots had the highest dominance factor (1.820) in all the data for their 16 game undefeated season in 2007. The next highest dominance factor in any sport was 1.696 by the Chicago Bulls for a 15 game win streak in 1997. Impressively, there were 8 streaks with a dominance factor of more than 1.5 in the NBA during the time period analyzed and all eight were achieved by the Bulls during the Michael Jordan era.

\section{Conclusions and Our Vote: Heat, Blackhawks, or Hawks?}

We have described two different measures for assessing the impressiveness of team streaks in baseball, basketball, football and hockey. The measures use gambling line data, which we feel are ideally suited to take into consideration all the factors that would affect the probability each team would win a particular game. We have computed the measures for all four sports and provided rankings within and across sports. The ranking of the streaks depends upon which measure is chosen and we believe the measures provide complementary insights. Statistical models that incorporate many game-specific factors and that are applied to specific games involved in streaks could be used in place of the gambling line information in the proposed measures. This would be an opportunity for future research.

Let's now take a closer look at the streaks of the Heat, the Blackhawks, and the Hawks and declare a winner (we won't be wishy-washy). These streaks are clearly among the most impressive streaks across all sports during the data period. The Blackhawks' streak has the best SP $(0.000074$ vs. 0.000269 for the Hawks and 0.000683 for the Heat), whereas the Heat's streak has the best ATSP ( 0.00000024 vs. 0.0000257 for the Blackhawks and 0.0000856 for the Hawks). The Heat's schedule during their streak was the easiest (schedule factor of 1.113 vs. 1.082 for the Hawks and 1.044 for the Blackhawks) but this is already taken into account in both measures. The Heat's dominance factor was much higher (1.358 vs. 1.199 for the Hawks and 1.045 for the Blackhawks) and this is the reason their SP is the lowest of the three but their ATSP is the highest (by far). In general, we think a team's dominance is part of what makes what they do impressive so we cast our vote for the Heat's streak as being the most impressive of these three recent streaks and, in fact, the most impressive of any streak in the time period for which we have data. For those that disagree, we have presented some quantitative

Table 3. Average dominance and schedule factors for streaks of 10 or more.

\begin{tabular}{cccc}
\hline Sport & Average Schedule Factor & Average Dominance Factor & Number of Streaks \\
NBA & 1.066 & 1.314 & 136 \\
NFL & 1.043 & 1.334 & 22 \\
MLB & 1.030 & 1.078 & 58 \\
NHL Winning & 1.014 & 1.111 & 13 \\
NHL Points & 1.048 & 1.032 & 77 \\
\hline
\end{tabular}


results that you are welcome to use to support your arguments.

\section{References}

[1] Mottley, C. (1954) Letter to the Editor-The Application of Operations-Research Methods to Athletic Games. Journal of the Operations Research Society of America, 2, 335-338. http://dx.doi.org/10.1287/opre.2.3.335

[2] Wright, M. (2009) 50 Years of OR in Sport. Journal of the Operational Research Society, 60, S161-S168. http://dx.doi.org/10.1057/jors.2008.170

[3] Cochran, J. (2005) OR Scores in Sports. OR/MS Today, 32, 32-40.

[4] Arbesman, S. and Strogatz, S. (2008) A Journey to Baseball's Alternate Universe. The New York Times, March 30.

[5] Rockoff, D. and Yates, P. (2009) Chasing DiMaggio: Streaks in Simulated Seasons Using Non-Constant At-Bats. Journal of Quantitative Analysis in Sports, 5, Article 4.

[6] Peel, D. and Thomas, D. (1992) The Demand for Football: Some Evidence on Outcome Uncertainty. Empirical Economics, 17, 323-331. http://dx.doi.org/10.1007/BF01206291

[7] Peel, D. and Thomas, D. (1988) Outcome Uncertainty and the Demand for Football: An Analysis of Match Attendance in the English Football League. Scottish Journal of Political Economy, 35, 242-249. http://dx.doi.org/10.1111/j.1467-9485.1988.tb01049.x

[8] Sauer, R. (1998) The Economics of Wagering Markets. Journal of Economic Literature, 36, 2021-2064.

[9] Gandar, J., Zuber, R. and Dare, W. (2000) The Search for Informed Traders in the Totals Betting Markets for National Baksetball Association Games. Journal of Sports Economics, 3, 133-148.

[10] Bowman, R., Ashman, T. and Lambrinos, J. (2013) Prospective Measures of Competitive Balance: Application to Money Lines in Major League Baseball. Applied Economics, 45, 4071-4081. http://dx.doi.org/10.1080/00036846.2012.750421

[11] Bowman, R., Lambrinos, J. and Ashman, T. (2013) Competitive Balance in the Eyes of the Sports Fan: Prospective Measures Using Point Spreads in the NFL and NBA. Journal of Sports Economics, 14, 498-520. http://dx.doi.org/10.1177/1527002511430230 


\section{Appendix}

Bowman, Ashman and Lambrinos [10] and Bowman Lambrinos, and Ashman [11] presented a conceptual model of the gambling spread (for any NFL or NBA game in any season) as:

$$
S_{j k}=N_{i j k}-H
$$

where

$S_{i j k}=$ the spread when team $i$ is playing at home against team $j$ in game $k$,

$N_{i j k}=$ the "neutral" spread—the spread adjusted by the average home advantage, and

$\mathrm{H}=$ the average home advantage (averaged across all games in the season).

They further broke down $N_{i j k}$ as follows:

$$
N_{i j k}=-\left(R_{i}-R_{j}+\varepsilon_{i j k}\right)
$$

where

$R_{i}=$ the rating of team $i$ (how much team $i$ would be favored against the average league team in a neutral site game based on the entire season — note that this means the average of all the ratings is 0 ), and

$\varepsilon_{i j k}=$ the factors specific to team $i$ playing team $j$ in game $k$.

They suggested estimating the team ratings using the following regression model with the $\varepsilon_{i j k}$ terms being the error terms in the model.

$$
S_{h(k) a(k) k}=\beta_{0}+\sum_{i=1}^{m-1} \beta_{i} X_{i}+\varepsilon_{h(k) a(k) k}
$$

where

$$
X_{i}=\left\{\begin{array}{cc}
-1 & \text { if } h(k)=i(\text { team } i \text { is the home team in game } k) \\
1 & \text { if } a(k)=i(\text { team } i \text { is the away team in game } k) \\
0 & \text { otherwise }
\end{array}\right.
$$

and $m=$ the number of teams in the league.

Note that one of the teams (team $\mathrm{m}$ ) must be left out of the model and that the coefficient estimates are relative to that team. To make the ratings estimates invariant to the team left out (and relative to the average team as required by the definition of the ratings terms), the coefficients are converted to the ratings as follows:

$$
\widehat{R}_{i}=\widehat{\beta}_{i}-\frac{1}{m} \sum_{j=1}^{m-1} \widehat{\beta}_{j}, i=1 \text { to } m-1 \text {, and } \widehat{R_{m}}=-\frac{1}{m} \sum_{j=1}^{m-1} \widehat{\beta}_{j} \text {. }
$$

Bowman, Ashman, and Lambrinos [10] and Bowman, Lambrinos and Ashman [11] showed how to translate this approach to the case where the gambling line information comes in the form of a money line. First, the money line is used to compute the probability that each team will win the game. Then 0.5 is subtracted from each team's win probability and the result is treated as a spread in the regression model just described.

The authors developed the ratings so that they could be used in competitive balance measures but we can use them to adjust the win probabilities in the manner necessary to compute ATSP. In the case where the ratings come from the win probabilities, the probability that the average team in the league would win any particular game in the streak would be the probability the actual team involved in the streak would win the game minus the rating for that actual team (since the average team would have a rating of zero). This new probability would then be adjusted the same as for SP if evaluating a point streak in hockey. If the gambling line information is in the form of a spread, the procedure just described would be used to adjust the spread to reflect the average team playing the game rather than the actual team. The win probabilities would then be computed the same as for SP but now based on the adjusted spread rather than the actual spread. 